\title{
Computer modeling of oxygen migration accompanying aluminum production
}

\author{
A. Y. Galashev ${ }^{\dagger}$, O. R. Rakhmanova \\ †alexander-galashev@yandex.ru
}

Institute of High-Temperature Electrochemistry, Ural Branch of RAS, Sofia Kovalevskaya Str., 22, Yekaterinburg, 620990, Russia

The behavior of oxygen ions in the Al melt under action of a constant electric field was studied by molecular dynamics. The rate of moving up of $\mathrm{O}^{2-}$ ions from the graphite wall to the melt surface increases and the time interval in which the first ion reaches the surface decreases with an increase in $\mathrm{O}^{2-}$ concentration. When the number of ions is less than 90 small ion clusters consisting of only a few oxygen ions are often formed on the Al melt surface. In the case when the number of ions is 90 , a vertical chain extending from top to bottom of the molecular dynamics cell is formed. The number of $\mathrm{O}^{2-}$ ions in the surface layer strongly depends on time. Before reaching the surface of the melts, an ion carries out a random motion in the vicinity of the bottom of the basic cell, and reaching the surface the ion moves randomly in a thin surface layer of the cell. Al atomic selfdiffusion coefficient and internal energy of the $\mathrm{Al}$ melts increase while $\mathrm{O}^{2-}$ ion selfdiffusion coefficient decreases with increasing the concentration of ions in the system. Modeling has shown that changing the boundary conditions in time can have a significant effect on the behavior of the "Al-O ${ }^{2-}$-graphite" system with a high content $(=90)$ of oxygen ions. Since the change in the boundary conditions is quite likely when aluminum melt enters the electrolyte, then during the smelting of aluminum, both relatively slow and rapid processes of oxygen release can occur.

Keywords: aluminum melts, molecular dynamics, oxygen ions, selfdiffusion, structure.

\section{Introduction}

Aluminum is the most abundant metal and the third most abundant element in the Earth's crust after oxygen and silicon. It makes up about $8 \%$ by weight of the Earth's solid surface. Aluminum is too reactive chemically to occur in nature as a free metal. Instead, it is found to be combined in over 270 different minerals. One of the minerals that is important in the production of aluminum metal is cryolite $\left(\mathrm{Na}_{3} \mathrm{AlF}_{6}\right)$. However, cryolite is not used as an ore because the aluminum is not extracted from it directly. To produce an electrolyte at lower temperature, alumina is dissolved in molten cryolite at $960^{\circ} \mathrm{C}$. A continuous $\mathrm{Al}_{2} \mathrm{O}_{3}$ layer can be formed on the surface of the electrolyte. In addition, low partial pressure of oxygen is observed in molten aluminum. The oxygen ions can be immersed into the molten aluminum because of a strong convection resulting in a strong evolution of gas $\left(\mathrm{CO}\right.$ and $\left.\mathrm{CO}_{2}\right)$ when aluminum is prepared using cryolite.

Unlike the extensive researches of oxydation of the solid $\mathrm{Al}$ phase, the role of aluminum melt and its influence on the trend of oxidation have not been taken into consideration yet [1]. The MD simulations of the oxidation of the carboncoated aluminum nanoparticles indicate that such particles were less reactive at low temperatures, but they became very susceptible to oxidation at higher temperatures when the coating layer was removed [2]. The initial oxidation of the bare Al substrate proceeds by an island-by-layer growth mechanism involving the lateral diffusion over the bare $\mathrm{Al}$ substrate surface of mobile oxygen species [3]. It was found that electric field causes an interstitial outward transport of $\mathrm{Al}$ cations through a close packing of $\mathrm{O}^{2-}$ anions in the amorphous films, and inward diffusion of $\mathrm{O}^{2-}$ along grain boundaries in the crystalline films respectively. For the electric field controlled Al cation motion, a value of $2.6 \mathrm{eV}$ was determined for the rate-limiting energy barrier which is located at the metal/oxide interface. The diffusion of oxygen anions in liquid aluminum is still poorly understood, although this phenomenon in the advanced aluminum smelting processes should cause considerable interest.

The chemical stability of the oxygen ion is lost when it meets an aluminum ion. However, for the primary ionization of an aluminum atom, an energy 37 times higher than the kinetic energy of the $\mathrm{Al}$ atom is required at $\mathrm{T}=1233 \mathrm{~K}$ (Al melting temperature). The formation of oxides of liquid melts is a complex physical-chemical phenomenon. This phenomenon has not been studied at the molecular level. Existing theoretical studies have rough assumptions [4,5]. It is usually assumed that the oxidation of an aluminum alloy takes place in the gas phase [6]. One side of the reaction front contains metal vapour, while the other contains only oxygen. Therefore, the concentration of oxygen in the liquid side of the reaction front is zero, while on the other side the metal concentration is zero. Strong oxidation of an aluminum alloy has been observed in the presence of elements like magnesium, sodium, lithium and zinc (single or in combination). 
Safety measures must be taken during the production of aluminum. Aluminum might impact indirectly the function of the nervous system and levels of cholesterol [7]. Acute exposure to aluminum dust impairs lung function, causes inflammation. Long-term exposure of aluminum could be deleterious and could worse synergistically the cognitive abilities of individuals. Sustainable impact of aluminum high level can cause the bone abnormalities. In connection with the existing negative effects on a person of various factors appearing in the production of aluminum, it is important to investigate this process at the molecular level. First of all, it is important to understand why the oxidative processes that reduce the risk factor are weakly expressed in aluminum melts.

In this article, we study the effect of the electric field on the behavior of oxygen anions during their movement through aluminum melts. We also consider the effect of the concentration of oxygen ions on their distribution in the volume of the melts and the possibility of the formation of a pop-up bubble on the surface of the melts in the presence of free boundary conditions.

\section{Model}

First, liquid system was prepared by melting FCC aluminum crystal at a temperature of $2000 \mathrm{~K}$ and density $\rho=2.55 \mathrm{~g} / \mathrm{cm}^{3}$. The basic cell of this system contained 4000 aluminum atoms. Periodic boundary conditions (PBC) acted along each Cartesian axis. After the Al crystal was completely melted the system was reduced by removing the upper half of the MD cell. By coordinate scaling the density of remaining system was brought to a value of $2.27 \mathrm{~g} / \mathrm{cm}^{3}$, which corresponds to $\mathrm{Al}$ melt at $1233 \mathrm{~K}$. Then $\mathrm{O}^{2-}$ ions are randomly (using a random number generator) inserted into the MD cell bottom part with thickness of $0.45 \mathrm{~nm}$. We check the distances between ions $\left(r_{\min }^{\mathrm{O} O}=0.3433 \mathrm{~nm}\right)$ and between ions and atoms of $\mathrm{Al}$ $\left(r_{\min }^{\mathrm{O}-\mathrm{Al}}=0.3026 \mathrm{~nm}\right)$. The values of chosen parameters $r_{\min }$ are equal to the $\sigma$ parameters of Lennard-Jones potential, which describes the relevant interactions. The $\mathrm{Al}$ atoms with the distances $r<r_{\min }^{\mathrm{O}-\mathrm{Al}}$ to the $\mathrm{O}^{2-}$ ions are removed from the system. The initial configuration was prepared containing different amounts of ions $N_{\text {oxygen }}$ (from 10 to 90) and the number of Al atoms (from 1731 to 1499). There is no electric field in a pure solid metal. Electrons are the charge carriers here. The aggregate state of metal affects its response to the applied electric field. There are no visible changes in the solid conductor in the presence of the external field. On the contrary, the motion of the metal ions in the liquid state leaves traces of the reaction to the action of the electric field. The flat boundary of the metal is unstable in a strong electric field. The development of instability is expressed in the presence of conical singularities, terminating with the sharp protrusions from which emission processes are initialized. In addition, there is a movement of liquid metal containing ions in vacuum under the action of alternating electric field. A liquid metal system that is strongly diluted with oxygen ions is investigated in this paper. Here the oxygen ions are the charge carriers. In any substance (including liquid metal) placed in the electric field its electric charges (ions $\mathrm{O}^{2-}$ ) are influenced by the external electric field $E_{1}$. Internal electric field $E_{2}$ arises in the substance in this case. The total electric field $E$ is summarized in accordance with the superposition principle from the external field $E_{1}$ and the internal one $E_{2}$ created by the charged particles of the substance. As a result, some of the charges in the system are moved in the necessary direction forming a current. A rigid impenetrating wall above the melt and limitation to the escape of atoms and ions from the top of the MD cell in the vertical direction are applied. A permanent electric field of $50 \mathrm{~V} / \mathrm{m}$ acts between electrodes represented by an impermeable wall (anode) and graphite (cathode). Such an order of electric field strength can exist during aluminum smelting.

The further calculations were made at a temperature of $1233 \mathrm{~K}$ with $\mathrm{PBC}$ operating in horizontal (ox and $o y$ ) directions. Two types of calculations at such boundary conditions were performed. There was not any gap between the top wall and the melt in the case of Al melting i.e. the melt's density could not be changed. Calculations of the second type were carried out after the first type ones using the same initial configurations as for calculations of the first type. In the second case, the rigid wall was moved away from the melts surface at a distance of $h=2.17 \mathrm{~nm}$, which corresponds to the vertical size of the cropped MD cell. Thus the possibility for $\mathrm{O}^{2-}$ ions to freely come out of the melt was provided. The lower part of the MD cell was limited by three-layer graphite substrate containing 2100 atoms. The equations of motion are integrated by the fourth-order Runge-Kutta method with the time step of $\Delta t=10^{-16} \mathrm{~s}$. The value of the time step is chosen empirically. Time step corresponds to such a value at which there is a chance to maintain the preset temperature of molten metal with high content of charged particles (ions). In fact, a small time step is used because of the high mobility of $\mathrm{O}^{2-}$ ions in the melt. It is substantially higher than the mobility of the metal atoms at the temperature of $1233 \mathrm{~K}$.

The Finnis-Sinclair (the generalized EAM) potential is used to describe $\mathrm{Al}-\mathrm{Al}$ interactions [8]. It is justified from the viewpoint of electron theory of a solid body. The total energy of an atom $i$ is given by

$$
V_{i}=F_{\alpha \beta}\left(\sum_{j \neq i} \rho_{\alpha \beta}\left(r_{i j}\right)\right)+1 / 2 \sum_{j \neq i} \phi_{\alpha \beta}\left(r_{i j}\right),
$$

where $F$ is the embedding energy which is a function of the atomic electron density $\rho, \rho$ is a functional specific to the atomic types of both atoms $i$ and $j$, so that different elements can contribute differently to the total electron density; $\phi$ is a pair potential interaction; $\alpha$ and $\beta$ are the element types of atoms $i$ and $j$, respectively. The multi-body nature of the Finnis-Sinclair potential is a result of the embedding energy term. This potential takes into account the temperature effects automatically because $\rho$ is a function of temperature according to definition.

The interatomic interactions inside layers of graphite were represented using the Tersoff many-body potential [9]. This potential is based on the concept of binding order. The potential energy between the neighboring atoms $i$, and $j$ is written as

$$
V_{i j}=f_{C}\left(r_{i j}\right)\left\lfloor A \exp \left(-\lambda^{(1)} r_{i j}\right)-B b_{i j} \exp \left(-\lambda^{(2)} r_{i j}\right)\right\rfloor,
$$




$$
f_{C}\left(r_{i j}\right)=\left\{\begin{array}{cc}
1, & r_{i j} \leq R^{(1)} ; \\
\frac{1}{2}+\frac{1}{2} \cos \left[\frac{\pi\left(r_{i j}-R^{(1)}\right)}{R^{(2)}-R^{(1)}}\right], & R^{(1)}<r_{i j}<R^{(2)} \\
0, & r_{i j} \geq R^{(2)}
\end{array}\right.
$$

where $b_{i j}$ is the many-body binding-order parameter describing the creation of the bond-formation energy (the attractive part $V_{i j}$ ) for the local atomic arrangement because of the presence of other neighboring atoms. The potential energy is a many-body function of the positions of atoms $i, j$, and $k$ and is determined by the parameters

$$
\begin{gathered}
b_{i j}=\left(1+\beta^{q} \xi_{i j}^{q}\right)^{-1 /(2 q)}, \\
\xi_{i j}=\sum_{k \neq i, j} f_{C}\left(r_{i j}\right) g\left(\theta_{i j k}\right), \\
g\left(\theta_{i j k}\right)=1+c^{2} / d^{2}-c^{2} /\left[d^{2}+\left(h-\theta_{i j k}\right)^{2}\right],
\end{gathered}
$$

where $\beta \cdot \xi$ is the effective coordination number, $g\left(\theta_{i j k}\right)$ is the function of the angle between $r_{i j}$ and $r_{i k}$, which stabilizes the structure of the tetrahedron.

The interlayer interactions in graphite $(\mathrm{C}-\mathrm{C})$ and $\mathrm{Al}-\mathrm{C}$, $\mathrm{C}-\mathrm{O}, \mathrm{Al}-\mathrm{O}$ interactions were described by Lennard-Jones potential. The interaction between oxygen ions was represented as the sum of Lennard-Jones and Coulomb contributions $\left(q_{0}=-2 e, e\right.$ is the elementary charge). Parameters of LennardJones potentials are taken from [10-12]. The Ewald summation was used for evaluating the long-range Coulomb interactions. Temperature was kept equal to given value by using Nose thermostat [13]. The total number of atoms and ions in the system was variable and consisted of no more than 4000 particles. At the same time, the interaction in different subsystems (molten metal and graphite) was described by many-particle potentials (EAM and Tersoff). A great amount of computer time is required to use such potentials. Besides, the process of forced diffusion of ions in a dense liquid medium is investigated at the atomic-atomic interaction of all melt components with the external boundary. Another reason for relatively small number of particles in the active part of the model is long calculation time. Usually one run of 5 million time steps lasted during 40 hours.

Selfdiffusion coefficient was calculated through the mean square displacement of the atoms $\left\langle[\Delta \mathbf{r}(t)]^{2}\right\rangle$

$$
D=\lim _{t \rightarrow \infty} 1 /{ }_{(2 \Gamma t)}\left\langle[\Delta \mathbf{r}(t)]^{2}\right\rangle,
$$

where $\Gamma=3$ is dimension of the space, the $\langle\ldots\rangle$ angle brackets denote time averaging.

A modified LAMMPS code [14] for parallel computing in the applied MD method was used. The calculations were performed on a hybrid cluster calculator "Uran" at IMM UB RAS with a peak performance of $216 \mathrm{Tflop} / \mathrm{s}$ and $1864 \mathrm{CPU}$.

\section{Results}

The initial configuration of the system in the form of three mutually conjugated cells (main and two images to the left and to the right along the axis $o x$ ) at $N_{\text {oxygen }}=90$ is shown in Fig. 1a. It is seen that the oxygen ions are randomly distributed all at the bottom of the cell and can be directly in contact with the top sheet of graphite. The lower part of the cell, where the ions coexist with a small number of $\mathrm{Al}$ atoms, has sufficiently a loose packing. There are clearly visible voids in irregular packaging of $\mathrm{Al}$ atoms in the volume of each cell. There are voids at the borders of the basic unit with its images. It was not possible for each ion to pass vertically through the thickness of the melt to the surface regardless the concentrations of oxygen ions. Each ion moves to find a space for itself, for example, to take a cavity in the melt. $\mathrm{Al}$ atoms, intensively interacting and moving quickly at a high temperature melt, eject ions and occupy the vacated space. In the presence of the external electric field there is a trend of upward lifting the ions in melt. However, the large concentration of charged particles in a confined space of the melt is not sustainable because of electrostatic repulsion. So, small ion clusters consisting of only a few $\mathrm{O}^{2-}$ ions are often formed. They may be formed in different parts of the MD cell volume. However, ultimately, their content on the melts surface still dominates. The increased content of oxygen ions on the melt surface at small $N_{\text {oxygen }}$ remains dynamically stable. However, with increasing $\mathrm{O}^{2-}$ concentration in the system a dynamic equilibrium is disturbed and ions on the surface begin looking for new places to locate. In the case where the number of ions $N_{\text {oxygen }}=90$, there is the most significant transformation of their location. If at the time of $400 \mathrm{ps}$ $52 \mathrm{O}^{2-}$ ions are on the surface of the melts then by the time of 500 ps there are only 18 ones in this area. A substantial portion of the ions (50) forms the vertical chain extending from top to bottom of the MD cell (Fig. 1b). The chain has a width of several ions and passes through the central part of the main MD cell face. Obviously, the boundary between the main MD cell and its periodic images is the weakest place in the melts where ions have rushed.

Fig. 2a represents the time $\tau_{1}$ of the first ion $\mathrm{O}^{2-}$ appearing on the surface of the molten metal in the vicinity of the top impermeable wall. Time $\tau_{1}$ consistently decreases as the $N_{\text {oxygen }}$ ions number in the system. As a result, by increasing the concentration of $\mathrm{O}^{2-}$ ions by factor 9 (from 10 to 90 ions) the $\tau_{1}$ time decreased 6 times. Reducing the $\tau_{1}$ time with increasing ion concentration, is primarily due to increased repulsion between ions with increasing $N_{\text {oxygen }}$, since the collision frequency is directly proportional to the number of ions. Minor role played in reducing $\tau_{1}$ is slight decrease of the system density with increasing $N_{\text {oxygen }}$. So due to the used method for generating initial configurations the system number density at $N_{\text {oxygen }}=90$ was reduced by $5 \%$ compared to the density at $N_{\text {oxygen }}=10$. The $\mathrm{O}^{2-}$ ions number $N_{\text {oxygen }}^{s}$ on the molten metal surface with thickness of $0.4 \mathrm{~nm}$ at the end of the MD calculation (at time $500 \mathrm{ps}$ ) is shown in Fig. $2 \mathrm{~b}$. The $N_{\text {oxygen }}^{s}$ distribution depending on the number of ions in the system has a maximum at $N_{\text {oxygen }}=50$. Obviously, at $N_{\text {oxygen }}=50$ such a concentration of $\mathrm{O}^{2-}$ ions is achieved when the electric field gets optimum support from the repulsive Coulomb interaction of ions to create their upward movement. A relatively stable over time the $\mathrm{O}^{2-}$ ions concentration is created in the surface melts zone in this case. In fact, the surface zone may comprise a much bigger number of $\mathrm{O}^{2-}$ ions 
but this number is not saved in time. For example, at time of $400 \mathrm{ps}$, the number of ions $\left(N_{\text {oxygen }}^{s}=53\right)$ in the surface region at $N_{\text {oxygen }}=901.8$ times bigger the value corresponding to the maximum of the distribution shown in Fig. 2b. However, after $100 \mathrm{ps}$ this value was lower (1.7 times) than the corresponding value relating to $N_{\text {oxygen }}=50$. In other words, when $t=400 \mathrm{ps}$ and $N_{\text {oxyen }}=90$ excessive number of ions is created in the surface region that leads to instability initiating the appearance of vertical oxygen filamentary formations. The average speed $v^{\perp}$ with which the ions reach the surface of the melt continuously increases with $N_{\text {oxygen }}$ (Fig. 2c). Eventually the $v^{\perp}$ value at $N_{\text {oxygen }}=90$ becomes higher than at $N_{\text {oxygen }}=10$ by factor 5.8. Such a large discrepancy in the lifting velocity of the ions appears due to the fact that at low concentrations of the majority of $\mathrm{O}^{2-}$ ions without having very frequent collisions with other ions diffuse into the Al melts volume. When the ion concentration becomes high enough their frequent collisions in conjunction with the lifting force of the electric field help to push $\mathrm{O}^{2-}$ anions to the melts surface region. Note that the most probable velocities $v_{m p}=\sqrt{2 \mathrm{kT} / \mathrm{m}}$ of the $\mathrm{Al}$ atoms and $\mathrm{O}^{2-}$ ions movement at a temperature of $1233 \mathrm{~K}$ are 871.7 and $1132.0 \mathrm{~m} / \mathrm{s}$, respectively. Thus, the magnitude of $v^{\perp}=812.0 \mathrm{~m} / \mathrm{s}$ at $N_{\text {oxygen }}=90$ approached the $v_{m p}$ value for $\mathrm{Al}$ but is still 1.4 times lower than the $v_{m p}$ value for $\mathrm{O}^{2-}$.

Previously, we have calculated the selfdiffusion coefficient of pure aluminum melt. In the framework of classical molecular dynamics we have shown that the calculated selfdiffusion and shear viscosity are sensitive to the choice of the density. Thus, at a temperature of $1200 \mathrm{~K}$, the values of $D_{\mathrm{Al}}=1.5 \cdot 10^{-8} \mathrm{~m}^{2} / \mathrm{s}$ and $1.3 \cdot 10^{-8} \mathrm{~m}^{2} / \mathrm{s}$ were obtained, which refer to the densities $2.18 \cdot 10^{3} \mathrm{~kg} / \mathrm{m}^{3}$ and $2.28 \cdot 10^{3} \mathrm{~kg} / \mathrm{m}^{3}$, respectively. The latter $D_{\mathrm{Al}}$ value agrees well with the experimental value $\left(1.28 \cdot 10^{-8} \mathrm{~m}^{2} / \mathrm{s}\right)$ obtained at the same density and temperature [15]. The $\mathrm{Al}$ atomic diffusion coefficient $D_{A_{1}}$ increases with the concentration of ions $\mathrm{O}^{2-}$ in the system (Fig. 3a). Increasing the mobility of $\mathrm{Al}$ atoms with a high content of oxygen ions appears due to

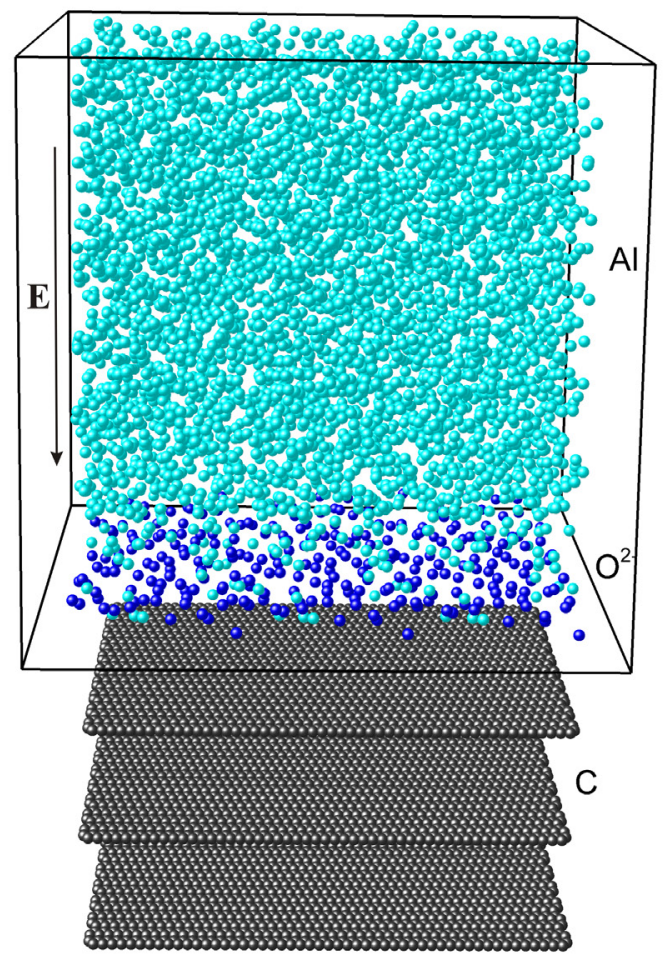

(a)

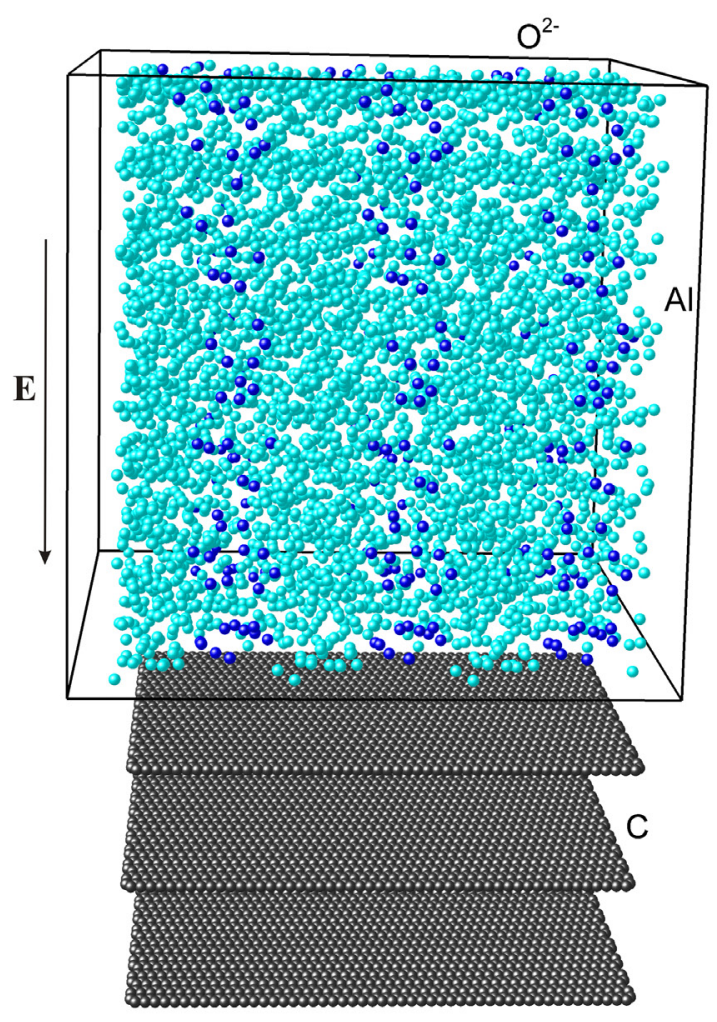

(b)

Fig. 1. Configurations of the "Al- $\mathrm{O}^{2-}$-graphite" system corresponding to the time points: (a) 0 ps, (b) of 500 ps, with the number of oxygen ions $N_{\text {oxygen }}=90$. The electric field strength of $50 \mathrm{~V} / \mathrm{m}$ has vertical direction (along the axis $o z$ ). Both the basic cell of the MD and its periodic images are presented along the $o x$ axis.
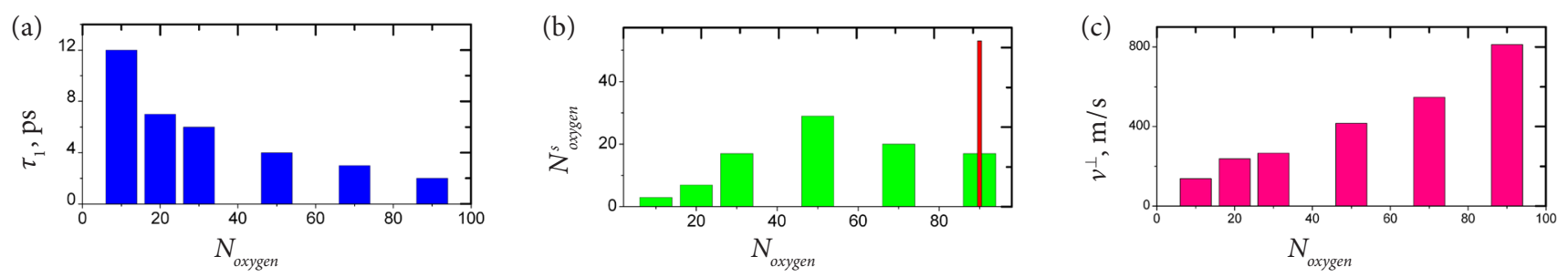

Fig. 2. Distributions (a) - (c) correspond to the relevant time 500 ps: (a) the time of the first ion reaching the melts surface; (b) the $\mathrm{O}^{2-}$ ions number on the molten metal surface with thickness of $0.4 \mathrm{~nm}$, narrow peak at the number of ions $N_{\text {oxygen }}=90$ corresponds to the time of $400 \mathrm{ps}$; (c) the average rate of the ions achievement of the melts surface. 
increased "friability" of the system when $N_{\text {oxygen }}$ is big. The dependence of the selfdiffusion coefficient $D_{\mathrm{O}^{2-}}$ of oxygen ions on the $N_{\text {oxygen }}$ number has the opposite character: the $\mathrm{O}^{2-}$ mobility decreases with increasing the concentration of ions (Fig. 3b). The lowest $D_{\mathrm{O}^{2-}}$ value is observed at $N_{\text {oxygen }}=90$. Despite a slight $D_{\mathrm{O}^{2-}}$ increase at $N_{\text {oxygen }}=30$, it again reduced when $N_{\text {oxygen }}=50$. Note that the value of the ion mobility by the order, and in some cases even two orders of magnitude higher than the mobility of $\mathrm{Al}$ atoms, which is associated with strong mutual Coulomb interaction of ions as well as the presence of a constant electric field. The $D_{\mathrm{O}^{2-}}$ decreases with an increase in the number of ions because of effect of crowding that slows fast-moving $\mathrm{O}^{2-}$ ions. A significant decrease (by almost 2 orders of magnitude) of the selfdiffusion coefficient of the $\mathrm{Al}$ atoms relative to that for pure aluminum melt is associated with an intensive motion of oxygen ions, which produces local melt densification and slows down the motion of $\mathrm{Al}$ atoms. There is no complexation in the "Al+ $\mathrm{O}^{2-"}$ system. The estimated value of the shear viscosity $\eta$ of a pure aluminum melt $(0.52 \mathrm{mPa} \cdot \mathrm{s})$, obtained according to the Stokes-Einstein relation: $\eta=k T /(6 \pi D R)$, where $R$ is the radius of the $\mathrm{Al}$ atom, is significantly lower than the experimental value $(2.17 \mathrm{mPa} \cdot \mathrm{s})$, not free of impurities at the same temperature [16]. This is mainly due to the use of a macroscopic description for phenomena occurring at the atomic level. The viscosity decreases with the addition of $\mathrm{O}^{2-}$ ions to the aluminum melt (see the inset in Fig. 3b) because the system becomes more friable, and the movement of $\mathrm{Al}$ atoms, which make up the vast majority, is freer.

To show the effect of obstacles (limited volume) for the $\mathrm{O}^{2-}$ ions escape from $\mathrm{Al}$ melts, we performed similar calculations with free boundary condition (FBC) acting in the direction of the $o z$ axis and PBC acting in the other two directions. In this case, the upper wall, moved a considerable distance, did not interfere with the motion of the ions. The same initial configurations as ones corresponding to the time $t=0$ in the calculations discussed above are used in these calculations. If the number of oxygen ions $N_{\text {oxygen }}<90$, the ions in each system behaved much like the case when PBC along the $o z$ direction operated. However, at $N_{\text {oxygen }}=90$ the situation changed dramatically: the pressure generated by the oxygen ions was enough to supplant the melts. In this case oxygen bubble began to expand and lift the melt unless the molten aluminum reached the bounding top wall (Fig. 4a). Furthermore, to determine how ions will behave in the system when they have the opportunity to move out through the upper surface of the melt, we continued our calculations of the first type, moving the top wall up to a distance $h=2.17 \mathrm{~nm}$.

For the systems with $N_{\text {oxygen }}<70$ we observed the removal from the melt of all ions occupying the surface region at time $t=500$ ps of the first calculation. The system "Al-O ${ }^{2-"}$ containing 70 ions is of particular interest. Ions located on the surface by the time of 500 ps left the melts. However, ions remaining in the depth of the melts, i.e. in the vicinity of the graphite bottom of the MD cells formed rapidly moving cluster changing its shape and strongly perturbing the whole melts. This excitement was detected on the surface of the melts also. In the system with $N_{\text {oxygen }}=90$ all ions including those located in the vertical chain move out through the upper surface of the melts (Fig. 4b). Configurations showed in Fig. 4a,b depict two alternative ways to implement the final state of the system with $N_{\text {oxygen }}=90$. In the first case oxygen is on the top of the MD cell and in the second case it is on its bottom.

\section{Discussion}

The change in the behavior of oxygen ions in the aluminum melt as a function of the oxygen concentration and the boundary conditions has been studied by molecular dynamics. The kinetic properties of the "O ${ }^{2-}-\mathrm{Al}^{\prime}$ system at a temperature of $1233 \mathrm{~K}$ were studied. The ions moved upward from the bottom of the MD cell to the surface of the melt under the action of a constant electric field. The event of reaching one of the ions of the surface of the Al melt was accelerated as oxygen ions were added to the melt. The average waiting time was reduced by 6.4 times with a change in the number of ions in the system from 10 to 90 . The average rate at which ions reach the surface of the melt increases as the number of ions increases and reaches $72 \%$ of the most probable velocity for $\mathrm{O}^{2-}$ ions at $T=1233 \mathrm{~K}$ in the absence of the electric field. An increase in the oxygen content in the system leads to the increase in the self-diffusion coefficients $\mathrm{D}$ of $\mathrm{Al}$ and to the decrease of that of $\mathrm{O}^{2-}$ ions. The viscosity of the gas-containing metal melt is reduced.

The oxygen ions form a chain along the applied electric field at a high concentration of ions in limited volume. The formation of the chains from oxygen ions also takes place in live nature. There is a certain analogy with the passage of the biological process of glycolysis. Although glycolysis is anaerobic, requiring no oxygen. Oxygen needs at the end of (a)

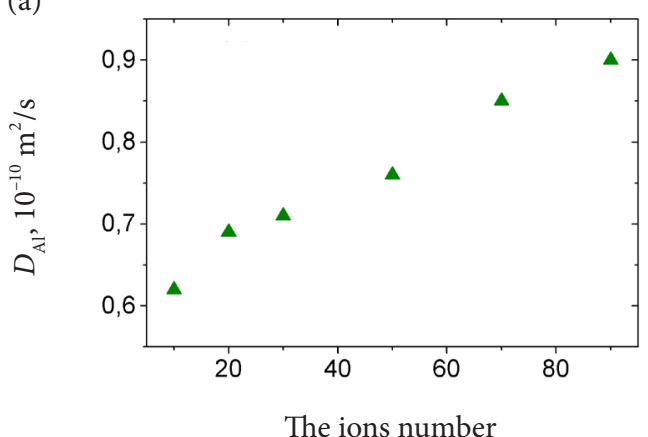

(b)

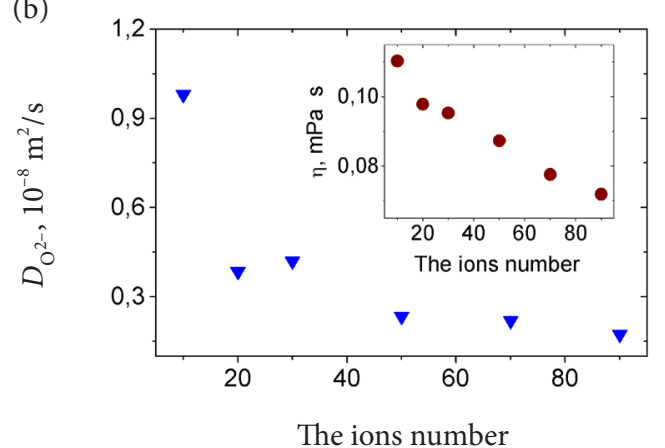

Fig. 3. The selfdiffusion coefficients: (a) $\mathrm{Al}$ and (b) $\mathrm{O}^{2-}$ depending on different contents of oxygen ions in the system "Al- $\mathrm{O}^{2-"}$ at $1233 \mathrm{~K}$. The insert represents the dependence of the shear viscosity of the $\mathrm{Al}$ melt on the content of $\mathrm{O}^{2-}$ ions in it. 


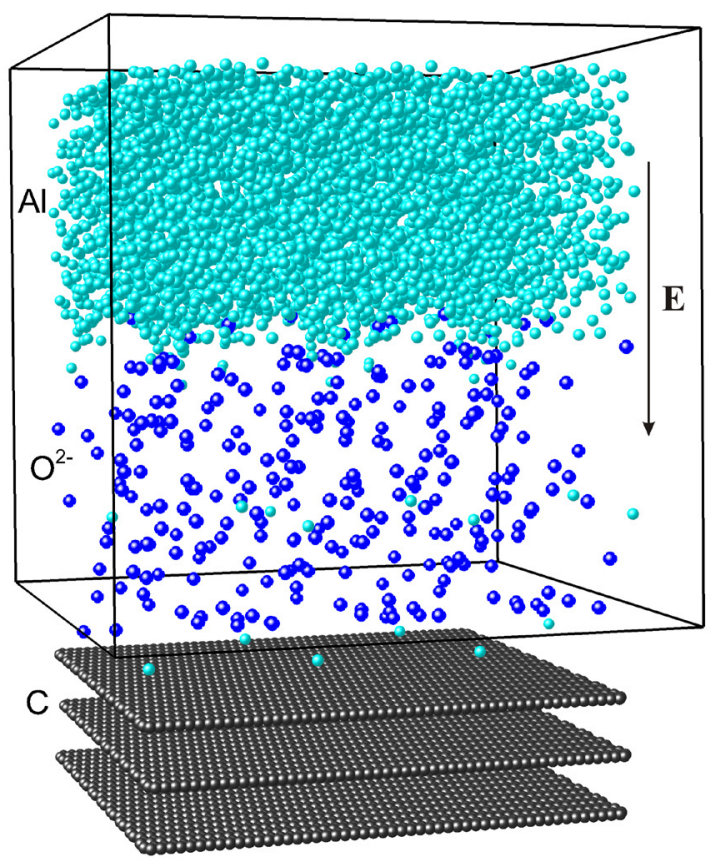

(a)

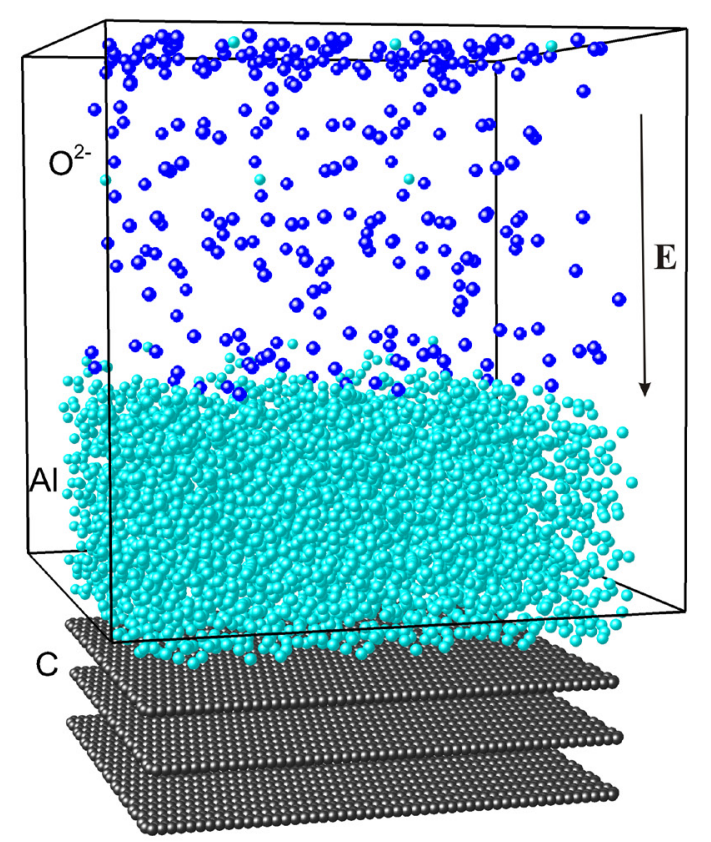

(b)

Fig. 4. The "Al-O $\mathrm{O}^{2-}$-graphite" system configuration obtained after a calculation of $200 \mathrm{ps}$ at the free boundary conditions along the $o z$ axis and PBC operating along $o x$ and $o y$ directions. The number of oxygen ions $N_{\text {oxygen }}=90$. The electric field strength of $50 \mathrm{~V} / \mathrm{m}$ has vertical direction (along the $o z$ axis). Initial configurations correspond to ones of the previously described calculations, when (a) $t=0$, (b) $t=500$ ps. The basic MD cell and its periodic images are presented along the $o x$ axis.

the electron transport chain, where it is the terminal electron acceptor.

The oxygen content in molten aluminum is not precisely determined but there are the measurements of the oxygen concentration in other metallic melts. The oxygen concentration in liquid lead, tin and copper were determined by a coulometric titration method [18-20]. The total quantity of electrical charge due to ion was defined by oxygen concentration in liquid. The oxygen activity in liquid antimony in the high oxygen partial pressure range was measured [21]. Authors reported the deviation from Henry's law for oxygen in liquid antimony. In addition, they pointed out that the oxide might be formed at the metal-electrolyte interface under their experimental conditions upon passing a current. The temperature dependence of oxygen solubility in a liquid lead-bismuth eutectic alloy was expressed by the following equation [22]

$$
\log \left(\mathrm{C}_{\mathrm{O}}\right)=-4.74 \times 10^{3} / T+7.06( \pm 0.03)
$$

$(878 \leq T \leq 1073)$

The electrons are delocalized in a solid metal. The metal is held together by the strong forces of attraction between the positive nuclei and the delocalized electrons. In a molten metal, the metallic bond is still present, although the ordered structure has been broken down. The metallic bond isn't fully broken until the metal boils [17]. We see that if the metal atoms are electrically neutral, the tendency to oxidation of the molten metal is not observed because of very rapid movement of oxygen ions in the melt volume in the presence of metallic bond.

\section{Conclusion}

Some of the ions entering the Al melt can behave differently depending on the concentration, on the layer thickness of molten metal over ions and on the number of negative ions at the border with the melt in the intended withdrawal site. If escape of ions from the melt into the electrolyte is readily, then depending on the concentration of oxygen the passage of ions through the electrolyte can pass slowly (by single and small groups), but can also occur the bubble oxygen output mode, which accompanied by disturbance of the melt surface and spraying the liquid Al. In presence of temporary obstacles to the output of ions from the melt at its boundary with the electrolyte, vertical chains of these ions can form. When removing the restrictions on the release of oxygen ions from the melt, jets of ions from the electrolyte can occur. This anomalous behavior of oxygen ions taking place when a high concentration of $\mathrm{O}^{2-}$ is contained in the $\mathrm{Al}$ melts disturbs the laminar motion of the ions in the electrolyte and creates the conditions for the emergence of electric breakdown. Spraying of the Al melts increases its solubility in the electrolyte and increases loss of metal produced by electrolysis. Furthermore, an extremely large number of rapid flows of ions directed to carbon anodes may cause a sharp increase in the formation of $\mathrm{CO}_{2}$ gas. In the case of $\mathrm{CO}_{2}$ film formation on the anode voltage of the operating cell increases to a large extent, and the gas ionic discharge breaks the film of gaseous $\mathrm{CO}_{2}$. The presence of oxygen ions in the air enhances the metabolism and increases the harmful effect of sprayed aluminum on a person. 
Acknowledgments. The work is executed with the financial support of Federal Target Program (agreement number 14.607.21.0146), unique identifier of the project RFMEFI60716X0146.

\section{References}

1. S. Hasani, M. Panjepour, M. Shamanian. Oxid. Met. 78, 179 - 195 (2012). DOI: 10.1007/s11085-012-9299-1

2. S. Hong, A.C. T. van Duin. J. Phys. Chem. C. 120, 9464 - 9474 (2016). DOI: 10.1021/acs.jpcc.6b00786

3. L.P. H. Jeurgens, W.G. Sloof, F.D. Tichelaar, E. J. Mittemeijer. J. Appl. Phys. 92, 1649 - 1656 (2002). DOI: $10.1063 / 1.1491591$

4. D. Krewski, R. A. Yokel, E. Nieboer, D. Borchelt, J. Cohen, J. Harry, S. Kacew, J. Lindsay, A. M. Mahfouz, V. Rondeau. J. Toxicol Environ Health B Crit Rev. 10, 1-269 (2007). DOI: 10.1080/10937400701597766

5. W.M. Zhong, G. L'Esperance, M. Suery. Metall. Mater. Trans. A. 26, 2625-2635 (1995). DOI: 10.1007/ BF02669420

6. P.N. Anyalebechi. Scr. Metall. Mater. 33, 1209-1216 (1995). DOI: 10.1016/0956-716X(95)00373-4

7. A. de Kanti, A. Mukhopadhyay, S. Sen, I.K. Puri. Modelling Simul. Mater. Sci. Eng. 12, 389-405 (2004). DOI: $10.1088 / 0965-0393 / 12 / 3 / 003$

8. M.I. Mendelev, D. J. Srolovitz, G. J. Ackland, S. Han. J. Mater. Res. 20, 208 - 218 (2005). DOI: 10.1557/ JMR.2005.0024
9. J. Tersoff. Phys. Rev. Lett. 61, 2879-2882 (1988). DOI: 10.1103/PhysRevLett.61.2879

10. Y. M. Kim, S.-C. Kim. J. Korean. Phys. Soc. 40, 293 - 299 (2002). DOI: $10.3938 / \mathrm{jkps} .40 .293$

11. R. B. Bird, W.F. Stewart, E.N. Ligthfoot. Transport Phenomena. New York, Wiley. (2002) 866 p.

12. A. E. Galashev. Tech. Phys. 59, $467-473$ (2014). DOI: 10.1134/S1063784214040112

13. S. A. Nosé. J. Chem. Phys. 81, $511-519$ (1984). DOI: 10.1063/1.447334

14. S. Plimpton. J. Comp. Phys. 117, 1-19 (1995). DOI: 10.1006/jcph.1995.1039

15. F. Kargl, E. Sondermann, H. Weis, A. Meyer. High Temp. High Press. 42, 3-21 (2013).

16. Y. Rosenfeld. J. Phys. Condens. Matter. 11, 5415-5427 (1999). DOI: 10.1088/0953-8984/11/28/303

17. A.E. Galashev, O.R. Rakhmanova. High. Temp., 52, 374- 380 (2014). DOI: 10.1134/S0018151X14030110

18. C.B. Alcock, T.N. Belford. Trans. Faraday Soc. 60, 822 - 835 (1964). DOI: 10.1039/TF9646000822

19. T.N. Belford, C.B. Alcock. Tran. Faraday Soc. 61, 443 - 453 (1965). DOI: 10.1039/TF9656100443

20. H. Rickert, H. Wagner. Electrochim. Acta. 11, 83-91 (1966). DOI: 10.1016/0013-4686(66)85009-0

21. S. Otsuka, Z. Kozuka. Met Trans B. 10, 565 - 574 (1979). DOI: $10.1007 / \mathrm{BF} 02662559$

22. A. Kishimoto, A. Wada, T. Michimoto, T. Furukawa, K. Aoto, T. Oishi. Met. Trans. B. 47, $122-128$ (2006). DOI: $10.1007 / \mathrm{BF} 02662559$ 